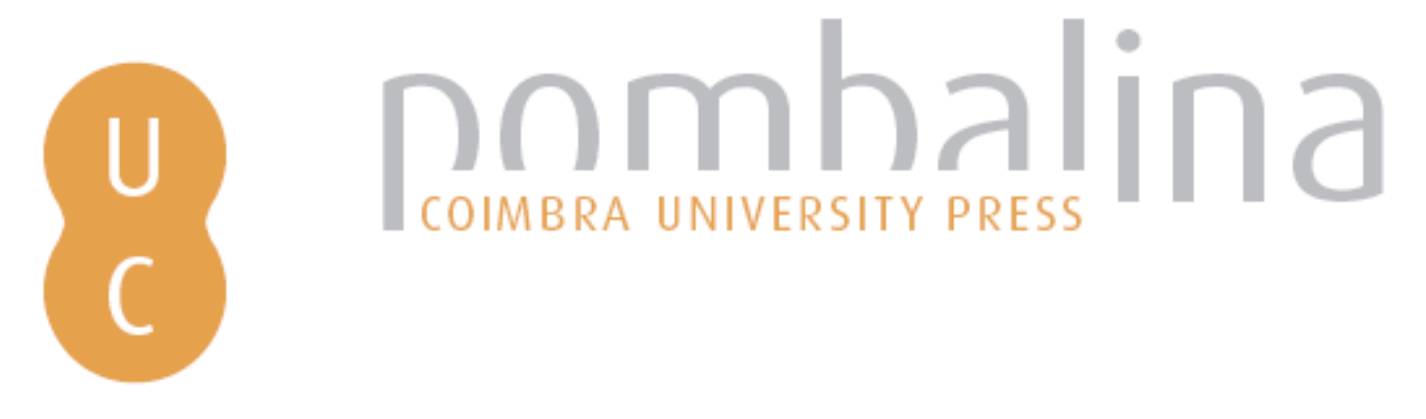

\title{
La régulation des comportements expressifs émotionnels
}
Autor(es):
Bitti, Pio E. Ricci

Publicado por: $\begin{aligned} & \text { Imprensa da Universidade de Coimbra; Les Éditions de L'IQRC (Les } \\ & \text { Presses de l'Université Laval) }\end{aligned}$

URL

persistente:

URI:http://hdl.handle.net/10316.2/32640

DOI:

DOI:http://dx.doi.org/10.14195/978-989-26-0805-1_8

Accessed : $\quad$ 26-Apr-2023 16:27:02

A navegação consulta e descarregamento dos títulos inseridos nas Bibliotecas Digitais UC Digitalis, UC Pombalina e UC Impactum, pressupõem a aceitação plena e sem reservas dos Termos e Condições de Uso destas Bibliotecas Digitais, disponíveis em https://digitalis.uc.pt/pt-pt/termos.

Conforme exposto nos referidos Termos e Condições de Uso, o descarregamento de títulos de acesso restrito requer uma licença válida de autorização devendo o utilizador aceder ao(s) documento(s) a partir de um endereço de IP da instituição detentora da supramencionada licença.

Ao utilizador é apenas permitido o descarregamento para uso pessoal, pelo que o emprego do(s) título(s) descarregado(s) para outro fim, designadamente comercial, carece de autorização do respetivo autor ou editor da obra.

Na medida em que todas as obras da UC Digitalis se encontram protegidas pelo Código do Direito de Autor e Direitos Conexos e demais legislação aplicável, toda a cópia, parcial ou total, deste documento, nos casos em que é legalmente admitida, deverá conter ou fazer-se acompanhar por este aviso.

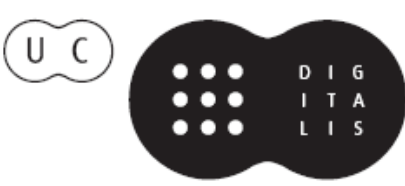


GILLES KIROUAC

Coordonnateur Scientifique

Cognition
et Émotions 
(Página deixada propositadamente em branco) 


\title{
GILLES KIROUAC
}

Coordonnateur Scientifique

\section{Cognition et Émotions}

\author{
AUTEURS \\ Klaus R. Scherer \& Janique Sangsue \\ Pierre Philippot \\ Dario Galati \\ Bernard Rimé \\ Pierre Gosselin \\ Ursula Hess \\ Arvid Kappas \& Jean Descôteaux \\ Pio E. Ricci Bitti \\ Pedro Luzes \\ Lise Fillion \\ Ramon Bayés \\ Coimbra - Imprensa da Universidade


COORDENAÇÃO EDITORIAL

Imprensa da Universidade de Coimbra

CONCEPÇÃO GRÁFICA

António Barros

PAGINAÇÃo

António Resende

[Universidade de Coimbra]

EXECUÇÃO GRÁFICA

G.C. - Gráfica de Coimbra, Lda.

Palheira - Assafarge - Apart. 3068

300I-453 Coimbra Codex

ISBN 972-8704-19-4

ISBN 2-7637-81।8-7

DEPÓSITO LEGAL 210430/04

(C) MAIO 2004, Imprensa DA UnIVERSIDAdE DE COIMBRA

\author{
OBRA PUBLICADA COM O PATROCINIO DO \\ INSTITUTO DE PSICOLOGIA COGNITIVA \\ FCT: FUNDAÇÃO PARA A CIÊNCIA E A TECNOLOGIA \\ MINISTÉRIO DA CIÊNCIA E DO ENSINO SUPERIOR \\ apolo do Programa Operacional CiênCIa, TeCnologia, Inovação \\ DO QUADRO COMUNITÁRIO DE APOIO
}


Pio E. Ricci Bitti

Département de Psychologie

Université des Études de Bologne

\section{LA RÉGULATION DES COMPORTEMENTS EXPRESSIFS ÉMOTIONNELS}

\section{Les émotions et leur régulation}

L'émotion représente chez l'homme une des expériences les plus significatives; en effet, elle accompagne l'individu tout le long de son existence pour lui fournir un modèle d'adaptation quant aux interactions entre l'organisme et le milieu. Tous sont désormais amplement d'accord sur le fait que l'émotion doit être considérée comme une structure psychologique à l'intérieur de laquelle interviennent diverses composantes (Scherer, 1982): une composante cognitive ayant pour finalité l'évaluation de l'événement-stimulus qui provoque l'émotion; une composante d'activation physiologique déterminée principalement par l'intervention du système neurovégétatif; une composante constituée par l'expression non-verbale; une composante motivationnelle, relative aux intentions et à la tendance à agir/réagir; une composante subjective consistant dans le sentiment éprouvé par l'individu. Toutes les composantes sont interdépendantes, synchronisées, et concourent à déterminer l'expérience émotionnelle, qu'il faut donc considérer comme un processus dynamique plutôt que comme une condition stable ou permanente [lzard 1977; Plutchik 1980; Scherer 1982].

Les auteurs contemporains continuent à être en désaccord quant à la définition des fonctions et à la signification des émotions: s'il prévaut le point de vue tenant les émotions pour des mécanismes essentiellement adaptatifs et motivationnels, mis à la disposition de l'organisme pour affronter le milieu ambiant, l'idée que l'émotion représente une sorte d'interférence ou «interruption» par rapport au déroulement de séquences comportementales, jouit encore d'un certain crédit. 
On peut considérer l'émotion comme un élément de médiation entre les événements environnementaux en perpétuel changement et les réponses comportementales de l'homme. Scherer [1982] indique trois aspects fondamentaux de ce processus de médiation: l'évaluation cognitive relevant de la stimulation ou de l'événement environnemental par rapport aux besoins, aux plans et aux préférences de l'organisme; la préparation physiologique et psychologique à l'action nécessaire pour affronter d'une manière adéquate la stimulation; la communication-signalisation transmise par l'organisme face au milieu environnant de son propre état, ses propres intentions et réactions.

L'évolution du système des émotions est relativement importante chez les espèces sociales: leur expression non verbale constitue une communication-signalisation pour les autres organismes qui peuvent en conséquence prévoir les comportements de l'individu qui expérimente l'émotion et planifier, à leur tour, les actions de façon appropriée.

L'analyse de l'expression et de la communication des émotions chez l'homme conduit à certaines considérations sur le problème de la régulation et du contrôle de ces émotions ainsi que sur les effets qu'une telle régulation provoque chez l'individu (cf. Fig. I).

La régulation est une composante essentielle du processus émotionnel: cela signifie que les individus ne se limitent pas à «éprouver» des émotions, mais que de façons différentes ils «interviennent» sur leur expérience émotionnelle; en fait, durant toutes les phases de cette expérience, et face à toutes les composantes du processus peuvent éventuellement être adoptées des actions régulatrices, contribuant à en moduler certains aspects. Pour pouvoir réaliser une régulation du processus émotionnel, l'individu doit être capable d'effectuer un «suivi» constant du processus en question, en fonction duquel il adoptera des stratégies spécifiques de régulation( ${ }^{(1)}$.

Presque tous les aspects de la réponse émotionnelle, on le sait, peuvent être soumis à des actions régulatrices, en ce sens qu'ils peuvent être, par exemple, inhibés, atténués, intensifiés, ou en tous cas modifiés dans leur expression.

(1) Une telle façon de définir la régulation des émotions laisserait à penser que l'acte régulateur est volontaire ; au contraire, il convient de rappeler qu'il n'existe pas de séparation absolue entre des processus involontaires et des stratégies volontaires de régulation. 


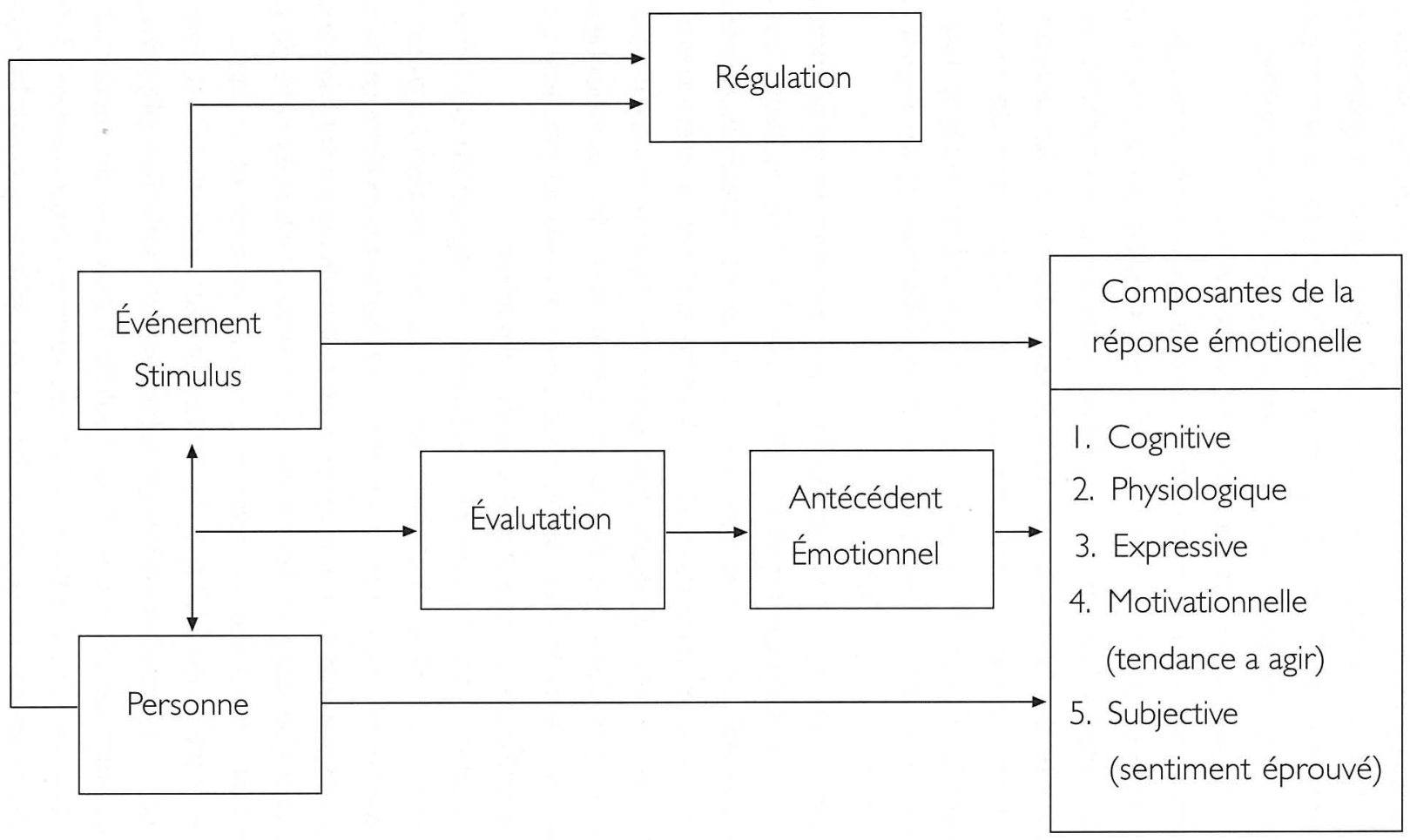

Figure I - Interdépendance entre les aspects de l'expérience émotionnelle 
Les objectifs des processus de régulation peuvent essentiellement être ramenés aux suivants: réduire ou augmenter les conséquences respectivement négatives ou positives des réponses comportementales induites par l'émotion et réduire le malaise ou accentuer le bien-être dérivant de l'expérimentation d'une certaine émotion. Dans le premier cas, on assiste à une régulation des résultats comportementaux de l'émotion, dans le deuxième, à une régulation intrapsychique des mécanismes d'évaluation.

La régulation peut donc concerner les processus cognitifs; nous pouvons citer à ce propos les mécanismes de défense dérivant de la psychanalyse, moyennant lesquels l'individu se «défend» contre une émotion dans la mesure où elle est désagréable ou porteur d'un état de tension non tolérable ou non approuvé socialement; il en est presque de même de certains aspects régulateurs des mécanismes de coping intrapsychique de Lazarus (1966) et les concepts d'évaluation défensive et de réévaluation constructive de Frijda (1986).

Les processus de régulation peuvent en outre intéresser les phénomènes d'activation physiologique, résultant de l'intervention du système nerveux autonome ou végétatif; un exemple très connu de ces stratégies régulatrices est représenté par les techniques du biofeed-back. D'autres mécanismes plus évidents et même plus analysés sont ceux de régulation mis en place à l'égard des expressions des émotions, c'est-à-dire des comportements observables qui découlent de la réponse émotionnelle. Ici, on fera surtout référence à cette partie des processus de régulation.

Par conséquent, nous pouvons considérer la régulation de l'émotion comme l'ensemble des stratégies adoptées par l'individu pour faire correspondre l'expérience intérieure et la manifestation extérieure de celleci aux situations sociales et aux normes socioculturelles qui y sont impliquées: il est donc clair que, dans un tel processus régulateur, interviennent de façon interdépendante des facteurs intérieurs, interpersonnels et sociaux.

$\mathrm{Si}$, d'une part, il est difficile de distinguer les interrelations entre le comportement expressif, l'expérience subjective et l'activation physiologique dans la régulation de l'émotion, il est toutefois plus aisé de reconnaitre le rôle des facteurs socioculturels; en effet, ces derniers interviennent à divers moments de l'expérience émotionelle: sur les stimuli qui, par le biais de l'apprentissage, sont en mesure de susciter des émotions précises, sur les règles qui en gouvernent la manifestation, sur la plupart des conséquences de l'activation émotionnelle [Ekman 1972; Scherer 1982]. 


\section{Exprimer les émotions et communiquer sur les émotions}

L'extériorisation des émotions, à savoir la modalité selon laquelle les émotions se manifestent extérieurement à travers la mimique faciale, les mouvements du corps, le langage et par le biais d'autres signaux plus ou moins repérables, a déjà fait depuis l'antiquité l'objet de nombreuses études. Cependant, ce n'est qu'avec Darwin (1872) que ces recherches se sont concentrées systématiquement autour de la fonction et de la signification de l'expression émotionnelle. Vues sous l'angle plus général du rapport homme-milieu, les réponses émotionnelles ont été tenues pour d'importants facteurs intervenant dans le but de réguler de manière adaptative la vie individuelle au sein d'un groupe social. L'expression et la reconnaissance d'états émotionnels déterminés permettent une plus grande capacité communicative entre l'individu et le groupe et garantissent une meilleure probabilité de survie à chacun d'entre eux. Pour appuyer cette thèse, on peut voir que seuls certains primates, en dehors de l'homme, possèdent un répertoire expressif au niveau de la mimique faciale suffisamment différenciée pour indiquer un certain nombre d'états émotionnels de base. Plus on monte dans l'échelle évolutive et examine des espèces vivant en communauté permanente, plus l'expressivité émotionnelle apparaît structurée et capable de communiquer des messages précis.

Ce n'est qu'à une époque relativement récente que les premières intuitions de Darwin ont été reprises par des éthologistes et par certains chercheurs tels que Ekman et Friesen (1969), lzard (197I), Tomkins (1980) et par d'autres ayant postulé l'existence de traits panculturels et universels dans l'expression faciale et la reconnaissance d'états émotionnels précis. D'autre part, on a vu (Ekman et Friesen, 1969) que, chez l'homme, contrairement aux primates, l'extériorisation des émotions peut, d'une certaine manière, être contrôlée intentionnellement. Ekman et Friesen (1969) ont parlé à ce propos des display rules, c'est-à-dire de règles d'exhibition influencées par les styles de comportement d'un groupe social déterminé. De plus, même si l'extériorisation des émotions représente un aspect capital de l'expérience émotionnelle chez l'homme, il faut dire que celle-ci interagit avec d'autres composantes de l'émotion, telles que, en l'occurrence, l'évaluation de la situation de stimulation, le vécu subjectif ou la disposition à agir. Ces autres composantes permettent de rattacher l'activité de l'expression non verbale des émotions à des aspects cognitifs et motivationnels particuliers. 
En d'autres termes, même si les réponses émotionnelles font partie du bagage biologique de l'être humain, elles sont suffisamment sophistiquées pour permettre à l'individu soit une évaluation de la situation extérieure en perpétuel changement, soit des vécus intérieurs. C'est-à-dire qu'elles permettent un rapport très souple avec le milieu.

Les éléments caractérisant l'extériorisation d'états émotionnels déterminés sont multiples et hétérogènes: parallèlement à une série de réponses physiologiques ou d'expressions faciales qui définissent de façon immédiate un type de réaction émotionnelle (comme la peur, la joie, la colère, la tristesse, la surprise ou le dégoût, pour rappeler celles que de nombreux spécialistes et auteurs mettent au rang des émotions fondamentales), il existe des messages (verbaux et non verbaux) qui servent à partager une expérience émotionnelle précise et d'en recueillir, entre les lignes, les nuances les plus infimes. Par conséquent, quand on parle de l'extériorisation des émotions, il peut être utile de faire une distinction entre expression et communication selon la nature du message extériorisé: à savoir, s'il est ou n'est pas intentionnel. En effet, ce n'est pas toujours que les aspects expressif et communicatif coïncident. Buck (1984) a proposé une subdivision entre la communication spontanée, propre à un système biologiquement partagé, se référant à un code d'origine génétique (pas appris) et la communication symbolique, propre à un système socialement partagé se rapportant à un code d'origine culturelle (appris). Pour Buck (1984) il existe donc un double code interprétatif qui délimite l'extériorisation des émotions et qui, de manière plus générale, représente sur un plan écologique le rapport entre l'homme et le milieu. L'aspect adaptatif de la communication spontanée se manifeste surtout dans le caractère non propositionnel de ce type de communication: aussi bien au niveau de la codification que de la décodification, les messages non

162 propositionnels donnent naissance à un rapport homme-milieu qui se précise dans un type de connaissance immédiate et dans une communication universellement partagée, ni volontaire, ni symbolique et ni même falsifiable. En revanche, la communication symbolique est caractérisée par un type de connaissance acquise par l'intermédiaire de messages propositionnels: elle est socialement partagée et fondamentalement intentionnelle et elle peut être falsifiable.

Reprenant ce qui a été énoncé plus haut au sujet du rapport entre expression et communication des émotions, on peut dire qu'à côté d'une 
fonction proprement adaptative (communication spontanée - expression des émotions) de l'extériorisation des émotions, il existe une fonction plus spécifiquement communicative, d'ordre symbolique, qui permet, si l'on se sert surtout d'instruments linguistiques, de communiquer sur les émotions, c'est-à-dire de partager un vécu émotionnel commun, en le réévoquant et le revivant ensemble. Tout ceci revêt une grande importance dans la détermination de ce qui touche aux effets sur l'individu mais aussi aux fonctions sociales ayant pour tâche de manifester extérieurement ses propres émotions.

\section{Styles de régulation et extériorisation émotionnelle}

Pour comprendre les effets des diverses modalités d'extériorisation des émotions, de nombreux chercheurs ont examiné le rapport entre les expressions faciales, plus facilement contrôlables, et les réponses physiologiques, involontaires de surcroît, dans le but de réussir à éclaircir quel est le poids qu'assument, au niveau de l'expression des émotions, les caractéristiques individuelles d'une part et d'autre part des situations sociales précises. Ces recherches se sont orientées en direction de deux axes théoriques. Le premier de ces modèles que l'on peut appeler celui de l'arousal met positivement en corrélation l'expression faciale avec l'activation physiologique (Lanzetta et al., 1976 ; Lanzetta et Kleck, 1970 ; Laird, 1974): l'expression émotionnelle proviendrait d'éléments variés qui agissent entre eux en synergie et se renforcent avec une série de feed-backs. Si l'on se réfère directement aux travaux de Darwin (1872), qui avait soutenu que l'expression émotionnelle intensifie l'expérience émotionnelle, de façon à ce que plus une émotion est exprimée, plus la sensation du sujet qui l'exprime est intense, ce modèle suit une hypothèse précise, connue sous le nom d' «hypothèse du feed-back facial» selon laquelle on suppose qu'un sujet exprimant librement et ouvertement ses propres émotions devrait avoir une réaction plus forte qui se traduirait aussi chez d'autres indicateurs émotionnels. En revanche, la deuxième théorie, qui a pris naissance à la suite des travaux de Jones (1935), a trouvé deux catégories de sujets - les intériorisateurs et les extériorisateurs - qui se différencient entre eux dans la mesure où les premiers présentent une expressivité faciale et mimique réduite ainsi qu'une haute réaction physiologique (électrodermale) tandis 
que les seconds présentent une forte expressivité extérieure et une faible activation physiologique. Ce modèle se rapproche du soi-disant modèle «cathartique», adopté tout du moins en partie par les théoriciens de la psychanalyse: dans ce cas, l'expression émotionnelle réduirait la tension intérieure. La corrélation inverse entre l'expressivité faciale et les réponses physiologiques constituerait une indication dans ce sens.

Si l'on se penche sur l'examen comparé des recherches ayant trait aux deux lignes théoriques mentionnées, il en émerge, d'une part, une nette confirmation de la discordance existant chez de nombreux sujets entre les indicateurs expressifs faciaux et les réponses physiologiques (Buck et al., 1972 ; Buck et al., 1974 ; Notarius et Levenson, 1979 ; Notarius et al., 1982; Field et Walden, 1982) et, d'autre part, dans les cas de forte anxiété, une activation générale des deux (Asendorpf et Scherer, 1983). Par contre, les confirmations spécifiques à l'hypothèse du feed-back facial (Buck, 1984) se sont avérées plus limitées. Même si les travaux de Field et Walden (1982) ont mis en évidence qu'il existait, dès la prime enfance, des styles expressifs différents du modèle intériorisation/extériorisation, il convient encore d'ajouter que les modes d'intériorisation et d'extériorisation peuvent être également appris en vertu de rôles sociaux déterminés, si bien qu'un individu peut agir selon une modalité intériorisatrice ou extériorisatrice en fonction de la situation où il se trouve.

On peut expliquer les discordances apparues au cours des travaux sur les modalités extériorisatrices et intériorisatrices, à propos des divers indicateurs des émotions par le fait que la dimension émotionnelle chez l'homme est organisée, on le sait, selon différents niveaux hiérarchiques, des plus archaïques aux plus évolutifs, et que chacun d'entre eux présente des systèmes divers de contrôle et de suivi des informations (Scherer, 1984). Si l'on considère l'hypothèse de Darwin relative au rapport entre expressivité et expérience émotionnelles, il faut analyser comment le passage de la sphère de l'expressivité à celle de l'expérience n'est pas automatique, mais s'exerce par l'intermédiaire d'une série d'éléments d'évaluation.

De nombreuses recherches sur les différents styles de régulation de l'activité émotionnelle effectuée par différents individus d'une part et d'autre part sur les effets que ces styles produisent sur ces mêmes individus à court et long terme ont mis en relief des typologies aux contours définis: Jones (1935), déjà cité, distingue deux catégories de sujet - les intériorisateurs et les extériorisateurs - qui se différencient entre eux dans la mesure où 
les premiers présentent une faible expressivité non verbale et une haute réaction physiologique, tandis que les deuxièmes présentent une forte expressivité extérieure et une faible activation physiologique; dans cette même ligne, Byrne (1964) a divisé en «repressors» et «sensitizers» les sujets extériorisant leurs émotions de différentes façons; la distinction traditionnelle proposée par Eysenk (1957) entre introversion et extroversion ainsi que la notion de «locus of control» extérieure et intérieure (Nowicki et Duke, 1974) sans oublier la «théorie de la polarité» (Tomkins, 1982) reproposent, elles aussi, même si elles le font selon des modalités diverses, une bipartition «intérieure-extérieure» à appliquer aux signaux émotionnels en fonction de leur «visibilité». || est important de noter comment tous ces modèles se réfèrent essentiellement à l'expression plutôt qu'à la communication des émotions. Parallèlement aux différences touchant les styles expressifs individuels, certaines typologies trouvent des réponses psychopathologiques, indices d'un équilibre altéré par rapport au milieu. II est intéressant de noter que c'est justement la polarité expressive (émotivité ; locus of control extérieur) qui est principalement impliquée dans les réponses de type pathologique. II semblerait donc que plus les émotions s'extériorisent, plus le bien-être psychophysiologique de l'individu fait défaut. En réalité tout ceci est vrai seulement si la prédominance du pôle expressif renvoie à une formation altérée du Soi, si bien qu'un individu n'est pas capable de contrôler ses propres vécus émotionnels ni de garder pour soi la «douleur mentale» qui en dérive (Bion, 1962).

D'autres recherches ont montré comment l'inhibition de la communication émotionnelle avait de profondes répercussions sur l'état de santé, surtout en présence de faits traumatisants. Pennebaker et O'Heeron (1984) ont fait voir comment les conjoints de personnes décédées à la suite d'un suicide ou d'un accident présentaient plus fréquemment des troubles de santé s'ils n'avaient pas pu ou pas voulu communiquer aux autres leurs émotions pendant le deuil. Cependant, contrairement aux autres recherches citées plus haut, celles-ci ont trait de façon prépondérante aux aspects relatifs à la communication symbolique des émotions: l'inhibition expressive coïncide dans ces cas-là avec l'impossibilité de communiquer à travers le langage (ou d'autres instruments symboliques non verbaux) l'expérience émotionnelle et, ce faisant, de l'élaborer. Rimé (1989) a signalé que la possibilité de revivre et évoquer à nouveau ses propres émotions protégeait contre les «effets à long terme» des événements traumatisants, alors qu'«à court terme» 
l'inhibition expressive pouvait atténuer la douleur indubitablement réveillée par le souvenir. Toutefois, même ici, il est bon de rester sur ses gardes et ne pas confondre les niveaux expressif et communicatif. Quand Rimé (1989) parle d' «effets à long terme», il entend surtout par là le dernier niveau: l'aménagement des souvenirs dans l'espace et le temps, appelé «déroulement» par l'auteur et correspondant à la condition préalable requise pour la reviviscence des émotions, consiste en pratique dans la création d'un univers symbolique. En revanche, pour ce qui est des «effets à court terme», ce sont surtout les aspects expressifs qui sont pris en considération (cf. Fig. 2).

La distinction entre les aspects expressifs et communicatifs au niveau de l'extériorisation des émotions est d'une importance cruciale dans le domaine clinique: l'incapacité à verbaliser ses émotions et dont de nombreux patients font preuve (trouble auquel Sifneos a donné le nom d'alexithymia, 1973, et que Marty et De M'Uzan,1963, ont défini «pensée opératoire») et qui, en grande partie, est étroitement liée à l'origine des troubles psychosomatiques, est tenue pour une véritable impossibilité d'élaborer des émotions et de construire son propre monde intérieur plutôt que pour une incapacité de type expressif (cf. Ricci Bitti e Gremigni, 1995; Ricci Bitti, Gremigni, Bertolotti, Zotti, 1995).

\section{Communication linguistique et élaboration symbolique de l'expérience émotionnelle.}

Pour pouvoir définir (et donc communiquer sur) son état émotionnel, il s'avère nécessaire d'avoir une certaine capacité d'auto-observation, une distance sûre entre le Soi et l'objet observé de façon à pouvoir considérer l'émotion vécue, même si on en reconnaît le caractère d'expérience

166 personnelle, comme autre par rapport à soi. À ce sujet, tant les travaux à caractère psychanalytique se référant au modèle des relations objectales, en particulier ceux de Winnicott (197I), que les contributions de spécialistes ayant décrit l'expérience émotionnelle en termes situationnels, surtout Frijda (1986) et Rimé (1989), illustrent très bien comment la possibilité de dénommer les émotions se rattache à la naissance et au développement des rapports interpersonnels. Dénommer les émotions favorise et soutient le lien avec l'autre: la capacité de s'observer et d'évaluer ses expériences émotionnelles fait partie intégrante du rapport interactif et permet d'éviter 


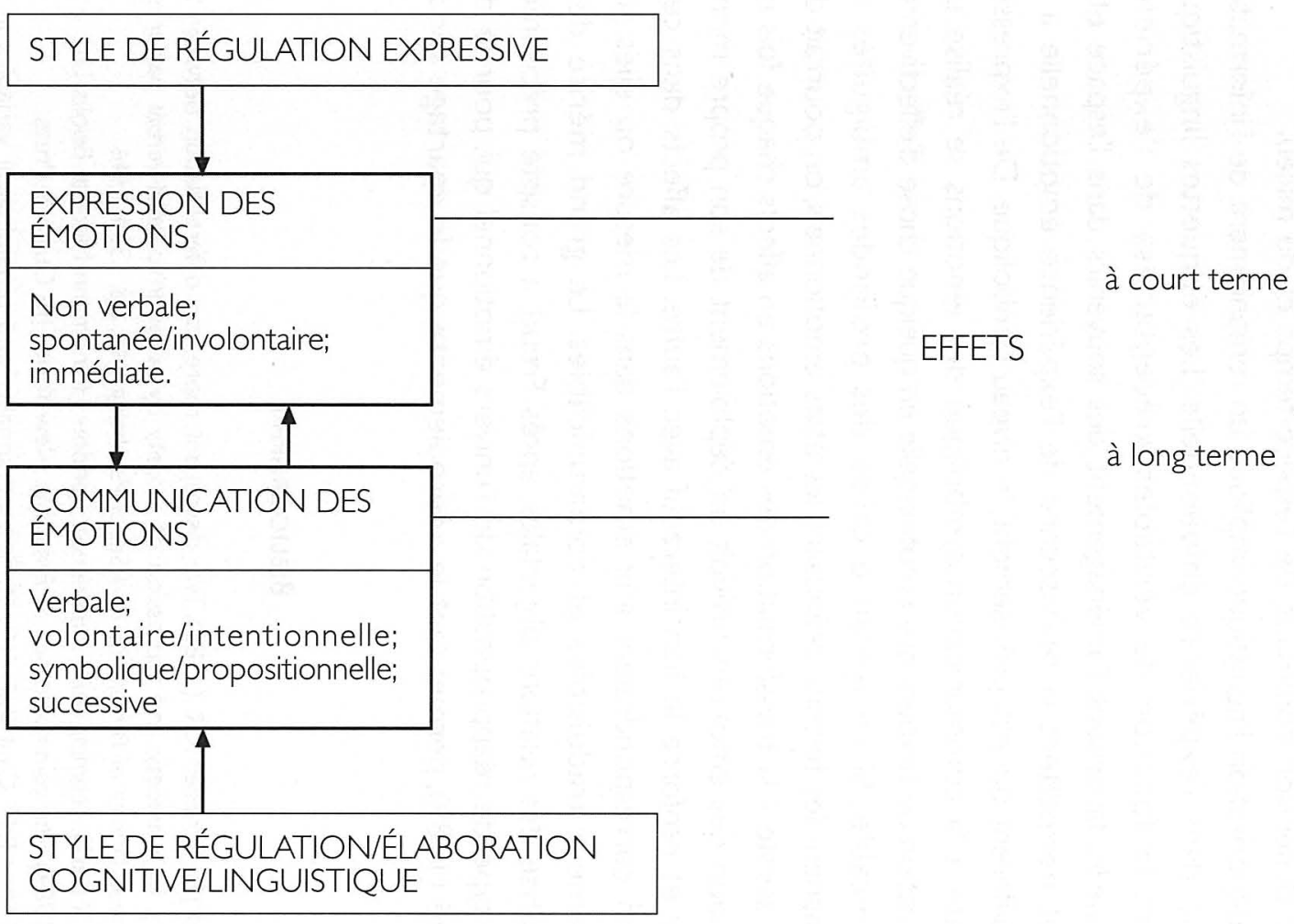

Figure 2 - Deux formes complémentaires d'extériorisation des émotions 
soit d'être excessivement bouleversé, soit de mettre une distance telle qu'il ne devient plus possible d'entrer en rapport avec l'autre. Donner un nom à ses émotions évite fondamentalement d'être écrasé au moment de leur impact, permet de créer des liens socio-affectifs et de retrouver, grâce à eux, une dimension commune de l'espace-temps et du milieu.

La catégorisation linguistique implique un remaniement de l'information contenue dans l'expérience émotionnelle. Les étiquettes linguistiques assument la fonction de véritables «réceptacles» de l'expérience émotionnelle, favorisent l'aménagement des souvenirs dans l'espace et le temps et permettent la reviviscence de l'expérience émotionnelle à un niveau différent qui est, précisément, le niveau symbolique. De l'expression immédiate à la communication symbolique des émotions se réalise une transformation de l'expérience émotionnelle en quelque chose d'effectivement communicable. Si ce n'était à cause des profondes ambiguïtés qui accompagnent les termes définissant les états émotionnels, on pourrait dire que l'on assiste à la transformation des émotions en affects chaque fois que l'élaboration des émotions prévoit un déploiement de son propre monde intérieur et renforce le lien interactif avec l'autre. Les affects dans cette acception correspondraient aux émotions dans la mesure où elles sont effectivement traduisibles et communicables. Le grand mérite de la psychanalyse des relations objectales, après Freud, a consisté précisément dans ce type de réappropriation de l'univers émotionnel qui, pour le dire à la Rimé (1989), permet tant le «déroulement» que le «partage social».

\section{BiBLIOGRAPHIE}

Asendorpf J.B., Scherer K.R. (1983). The discrepant repressor: differentiation between low anxiety, high anxiety, and repression of anxiety by autonomic-facial-verbal patterns of behaviour. Journal of Personality and Social Psychology. 45, pp. I334- 1346.

Bion W:R: (1962). Learning from Experience. London: Heinemann Medical Books Ltd.

Buck R: (1984). The communication of Emotions. New York: The Guilford Press.

Buck R., Miller R.E., Caul W.F. (1974). Sex, personality and physiological variables in the communication of affect via facial expression. Journal of Personality and Social Psychology. 30, pp. 587-596.

Buck R., Savin V.J., Miller R.E., Caul W.F. (1972). Communication of affect through facial expression in humans. Journal of Personality and Social Psychology. 23, pp. 362-37l.

Byrne D. (1964). Repression-sensitization as a dimension of personality. In: B.A. Maher (Ed.) Progress in experimental personality research (vol. I), New York: Academic Press. 
Darwin C. (1872). The Expression of Emotions in Man and Animals. London: Murray.

Ekman P. (1972). Universals and Cultural Differences in Facial Expressions of Emotion. In: J.

Cole (Ed.), Nebraska Symposium on Motivation 1971, Lincoln, Nebraska University Press.

Ekman P., Friesen W.V. (1969). The Repertoire of Nonverbal Behavior. Semiotica. I, pp. 49-98.

Eysenck, H.J. (1957). Dimensions de la personnalité, Paris: P.U.F.

Field T., Walden T. (1982). Production and perception of facial expressions in infancy and early childhood. In: H. Reese, L. Lipsitt (Ed.), Advances in child development (vol. 16), New York: Academic Press.

Frijda N.H. (1986). The Emotions. New York: Cambridge University Press.

Izard C.E. (197I). The face of emotion. New York: Appleton-Century-Crofts.

Izard C.E. (1977). Human Emotions, New York, Plenum Press.

Jones H.E. (1935). The galvanic skin response as related to overt emotional expression. American Journal of Psychology. 47, pp. 241-25I.

Laird J.D. (1974). Self-attribution of emotion: the effects of expressive behaviour on the quality of emotional experience. Journal of Personality and Social Psychology. 29, pp. 475-486.

Lanzetta J.T., Cartwright-Smith J., Kleck R.E. (1976). Effects of nonverbal dissimulation on emotional experience and autonomic arousal. Journal of Personality and Social Psychology. 33, pp. 354-370.

Lanzetta J.T., Kleck R.E. (1970). Encoding and decoding of nonverbal affect in humans. Journal of Personality and Social Psychology. 16, pp. $12-19$.

Lazarus R.S. (1966). Psychological stress and coping process. New York: McGraw-Hill.

Marty P., M'Uzan M. de (1963). La pensée opératoire. Revue Française de Psychanalyse. 27 (numéro spécial), pp. | 345- 1356.

Notarius C.I., Levenson R.W. (1979). Expressive tendencies and physiological response to stress. Journal of Personality and Social Psychology. 37, pp. 1204-1210.

Notarius C.I., Wemple C., Ingraham L.J., Burns T.J., Kollar E. (1982). Multichannel responses to an interpersonal stressor: interrelationships among facial display, heart rate, self-report of emotion and threat appraisal. Journal of Personality and Social Psychology. 43, pp. 400-408.

Nowicki S., Duke M.P. (1974). A locus of control measure for non-college as well as college educated adults. Journal of personality assessment. 38, pp. I36-137.

Pennebeker J.W., O'heeron R.C. (1984). Confinding in others and illness rates among spouses of suicide and accidental-death victims. Journal of Abnormal Psychology. 93, pp. 473-476.

Plutchik R. (1980). Emotion: A Psychoevolutionary Synthesis. New York: Harper and Row.

Ricci Bitti P.E., Gremigni P. (1995). Regolazione delle emozioni e rischio cardiovascolare. In: M. D'Alessio, P.E. Ricci Bitti, G.Villone Bettocchi (a cura di), Gli indicatori psicologici e sociali del rischio (pp. 301-318). Napoli: Gnocchi.

Ricci Bitti P.E., Gremigni P., Bertolotti G., Zotti A.M. (1995). Dimensions of Anger and Hostility in Cardiac Patients, Hypertensive Patients, and Controls. Psychotherapy and Psychosomatics. 64, pp. $162-172$.

Rimé B. (1989). Le partage social des émotions. In: B. Rimé et K.R. Scherer (direction de), Les émotions. Neuchatel: Delachaux et Niestlé.

Scherer K.R. (1982). Emotion as a Process: Function, Origin and Regulation. Social Science Information. 21, pp. 555-570.

Scherer K.R. (1984). On the nature and function of emotion: a component process approach. In: K.R. Scherer, P. Ekman (Eds.), Approaches to emotion. Hillsdale, N.J.: Erlbaum. 
Sifneos P.E. (1973). The prevalence of alexithymic characteristics in psychosomatic patients. Psychotherapy and Psychosomatics. 22, pp. 65-70.

Tomkins S.S. (1980). Affect as amplification: Some modification in theory. In: R. Plutchik, H. Kellerman (Eds.), Emotion, l: Theories of emotions. New York: Academic Press.

Tomkins S.S. ( 1 982). Affect Theory. In: P. Ekman (Ed.), Emotion in the human face. New York: Cambridge University Press.

Winnicott D.W. (1971). Playing and reality. London: Tavistock Publications. 
(Página deixada propositadamente em branco) 
Série

Investigaçāo

Coimbra

Imprensa da Universidade

Les Presses de L'Université Laval

Les Editions de L'IORC

2004 